\title{
Ion Trap Mass Analysis at High Pressure: A Theoretical View
}

\author{
Wei $\mathrm{Xu}^{\mathrm{a}}{ }^{\mathrm{Q}}$ Qingyu Song, ${ }^{\mathrm{b}}$ Scott A. Smith, ${ }^{\mathrm{b}}$ William J. Chappell, ${ }^{\mathrm{a}}$ and \\ Zheng Ouyang, \\ ${ }^{a}$ Department of Electrical and Computer Engineering, Purdue University, West Lafayette, Indiana, USA \\ ${ }^{\mathrm{b}}$ Department of Chemistry, Purdue University, West Lafayette, Indiana, USA \\ ${ }^{c}$ Weldon School of Biomedical Engineering, Purdue University, West Lafayette, Indiana, USA
}

The mass-selective manipulation of ions at elevated pressure, including mass analysis, ion isolation, or excitation, is of great interest for the development of mass spectrometry instrumentation, particularly for systems in which ion traps are employed as mass analyzers or storage devices. While experimental exploration of high-pressure mass analysis is limited by various difficulties, such as ion detection or electrical discharge at high-pressure, theoretical methods have been developed in this work to study ion/neutral collision effects within quadrupole ion traps and to explore their performance at pressures up to 1 Torr. Ion trapping, isolation, excitation, and resonance ejection were investigated over a wide pressure range. The theoretically calculated data were compared with available experimental data for pressures up to $50 \mathrm{mTorr}$, allowing the prediction of ion trap performance at pressures more than 10 times higher. (J Am Soc Mass Spectrom 2009, 20, 2144-2153) (c) 2009 American Society for Mass Spectrometry

$\mathrm{T}$ The exploration of mass analysis at high-pressure using electrodynamic devices, such as rf-driven quadrupole filters and ion traps, is of great interest in the development of mass spectrometers. In ion trap mass spectrometers equipped with an atmospheric pressure ionization source, a multi-stage differential vacuum system is usually used to transfer ions from atmospheric pressure to the mass analyzer which functions under vacuum (typically a few mTorr or less) [1-7]. The achievement of mass analysis at higher pressures would allow simpler vacuum systems with fewer differential pumping stages and higher ion transfer efficiencies. Ion traps, especially 2D linear ion traps, have been widely used in hybrid instruments as the trapping devices for reactions in front of the final stage for mass analysis [8-10]. rf-Driven ion optical components, such as the quadrupole (or "square quad"), are commonly used as ion guides over various pressure ranges [11-13]. Under appropriate operating conditions, such devices could potentially be used as linear traps or quadrupole filters to perform mass-selective operations, either alone or in tandem with other mass analyzers.

For portable instruments, the enablement of mass analysis at high pressures would have a large impact on the size and mechanical robustness of the vacuum system [14-18]. For example, mass analysis at tens of

Address reprint requests to Professor W. J. Chappell, Department of Electrical and Computer Engineering, Purdue University, West Lafayette, IN 47907, USA. E-mail: chappell@purdue.edu and Professor Z. Ouyang, Weldon School of Biomedical Engineering, Purdue University, West Lafayette, IN 47907, USA. E-mail: Ouyang@purdue.edu
mTorr would allow the use of drag pumps instead of turbo pumps. The recent development of the discontinuous atmospheric pressure interface (DAPI) represents a revolutionary step toward simpler, higher-pressure instrumentation. It allows a relatively low-power vacuum system to trap ions at high-pressure (100s of mTorr) under open-to-atmosphere conditions before sealing the vacuum for lower-pressure mass analysis (this at the cost of duty cycle) [19]. Despite this advance, much is still to be done toward the goal of low-power mass spectrometers. In particular, the increase of the mass analysis pressure would significantly increase the duty cycle and helps to minimize ion losses due to gas expansion [7].

The effects of pressure on ion trap performance have been extensively studied at pressures of several mTorr or lower. The collisions between ions and buffer gas molecules in an ion trap reduce the ion kinetic energy and, hence, the trapping efficiency during ion introduction is increased [20-24]. The cooling of the ions to the center of the trap also helps to improve mass resolution and mass accuracy by minimizing the effects due to difference in initial conditions for the mass selective instability scan $[8,25,26]$. The fragmentation of the ions for tandem mass spectrometry also relies on their collisions with the buffer gas molecules, which can cause increased ion internal energies and lead to subsequent fragmentation [27-31]. For each of these steps, dedicated experimental conditions are used to reach a balance between providing adequate collision energies and minimally interfering with intentionally stable ion trajectories. Helium is most commonly used as the 
buffer gas [22], with a pressure of several mTorr, although nitrogen and air have been used as buffer gases for linear ion traps [16, 32, 33]. An experimental exploration of ion trap operation at pressures higher than a few mTorr has been done and was recently reported in a separate manuscript [34]. Here, we use theoretical methods to study the fundamental role of pressure in ion trap mass analysis and to predict the potential of performing mass analysis at pressures much higher than those currently used.

The theoretical studies to characterize ion trap operations as a function of pressure have been carried out using simulation and/or theoretical modeling. In simulations, hypothetical scenarios are created in which trapped ions experience realistic features of an ion trap electrical field and operating conditions, which are close to experiments [4, 5, 35-41]. Discrete ion-molecule collision $[4,39,41]$ and space charge effects $[35,36]$ are included in the simulation settings. The simulation approach is especially powerful for system optimization and fine feature tuning. On the other hand, theoretical modeling provides relatively general and better understandings of the fundamental mechanisms behind phenomena [23, 42-55]. Ion motion in the presence of buffer gas has been investigated theoretically by solving the ion motion differential equation with the background buffer gas treated as a damping term [53]. In the pressure regions below several mTorr, discrete ionmolecule collision models [4, 41, 49] are preferred. However, damping term treatments for pressure effects have been shown to be effective at relatively high pressures for characterizing ion trap performance, including mass resolution $[45,55]$, the stability diagram [47], and ion nonlinear resonance phenomena [46, 56].

In this study, with the focus on ion trajectories at pressures much higher than several mTorr, the methods were developed based on the ion motion differential equation expressed with the pseudo-potential well approximation [51] and a damping term [53] as a representation of the buffer gas pressure. Aspects of ion trap performance at high pressures, such as ion trapping efficiency, ion isolation efficiency, and mass resolution during resonance ion ejection, have been investigated using the theoretical models. Consequently, a better understanding of ion-molecule collision effects during various ion trap operations was gained and predictions of the ion trap's performances at high pressures have been made.

\section{Modeling Method}

The ion motion differential equation was solved for high pressures and optimized AC resonance ejection conditions by following the treatments previously demonstrated by Major and Dehmelt [51], Goeringer et al. [45], and Arnold et al. [55]. Ion trajectory envelopes were calculated from the solution of the ion motion equation with the ion cloud initial condition treated as a random variable with Gaussian distribution. This theoretical model provides fundamental insights into the effects of the ion trap's AC resonance ejection optimization, the rf sweeping speed and frequency, the ion trap dimensions, the buffer gas pressure and the random nature of the initial ion conditions during trapping, ejection and isolation. The model accuracy was confirmed by comparison with experimental data [34] over the pressure range of several to $50 \mathrm{mTorr}$ using air as the buffer gas. On this basis, predictions were made for ion trap performance at higher pressures, where the experimental setup was limited by the potential electrical discharge and poor lifetime of the electron multiplier detector at high pressures. This model is expected to provide valid predictions for ion motion in a rf field for pressures up to 1 Torr. A more detailed discussion is provided below after the method is presented.

A 2D linear ion trap with center-to-electrode distances $\left(\mathrm{x}_{0}\right.$ and $\left.\mathrm{y}_{0}\right)$ of $5 \mathrm{~mm}$ and a length $\left(\mathrm{z}_{0}\right)$ of $40 \mathrm{~mm}$ was used as a model for the calculation. High order fields were not considered since the effects due to collisions were expected to be much more significant than those due to high order fields when an ion trap is operated at a pressure much higher than 1 mTorr. An rf frequency of $1.1 \mathrm{MHz}$ was used in the calculation.

\section{Ion Trapping}

Ion trapping is the first step of ion trap mass analysis. The analyte ions are either generated inside the ion trap or externally generated followed by injection into the ion trap with a certain kinetic energy, normally a few electron volts [26]. In a 2D ion trap, the trapping potential well along the long trap axis (commonly known as the $\mathrm{z}$ coordinate) is a DC potential well when balanced dipolar rf is applied between the rf electrode pairs. During injection, ions along the trap axis experience collisions with buffer gas molecules, lose kinetic energy, and are subsequently trapped by the z-axis DC potential well in addition to the rf pseudo-potential well in the $x-y$ plane.

The collision effect due to the ion-molecule collisions can be estimated using Langevin's collision theory [41, 49] and an elastic collision model. Based on Langevin collision theory, the mean free path of an ion in a buffer gas with electron polarization $\left(\alpha_{e}\right)$ and pressure $(P)$ is $[41,49]$

$$
\lambda=\frac{\sqrt{2 \mu} \varepsilon_{0} v R T}{e \sqrt{\alpha_{e}} N_{A} P}
$$

where $\mu$ is the reduced mass of the ion/neutral pair, $\varepsilon_{0}$ is the permittivity of air, $v$ is the speed of the ion, $R$ is the universal gas constant, $T$ is the temperature of the system, and $N_{A}$ is the Avogadro constant. Based on the elastic collision model, the kinetic energy loss ratio of the ion for each collision can be estimated as [57] 


$$
\gamma=\frac{K e^{\prime}}{K e}=\left(\frac{m-m_{b}}{m+m_{b}}\right)^{2}, \text { with } m_{b}<<m
$$

where $K e$ and $K e^{\prime}$ are the ion kinetic energy before and after the collision, $m$ is the mass of the ion and $m_{b}$ is the mass of the buffer gas molecule. Note that the buffer gas temperature is relatively low so that the buffer gas thermal velocity is much lower than the ion velocity hence buffer gas velocity can be ignored in this expression.

When ions are injected axially into a 2D ion trap operated with balanced rf and static voltages on its end electrodes, at least one ion-molecule collision is required to trap each ion. A method of reflecting the ions using higher voltage applied on the back end electrode was previously used to double the length of the ion path inside a trap [58]. As the ion kinetic energies are collisionally damped and their distribution becomes narrower, a greater abundance of the ions can be trapped by the z-axis DC potential well. For an ion with an initial kinetic energy (KE) $E_{0}$ (corresponding to a velocity of $v_{0}$ ) entering an ion trap with a pressure of $P$, the mean free path can be calculated using eq 1. A collision is assumed after the ion travels a distance of the mean free path and the kinetic energy loss can be calculated using eq 2. A new mean free path can then be calculated with the reduced speed $v$ and used for the calculation of further kinetic energy lost in subsequent collisions. Such a method was used to calculate the kinetic energy loss at various pressures for ions traveling along the axis of a 2D ion trap in a reflection mode [58] where a large DC voltage is applied to the back end electrode (travel path $=2 \mathrm{z}_{0}=80 \mathrm{~mm}$ ). Figure 1a shows the KE losses for ions with initial KEs of 10, 30, and 50 $\mathrm{eV}$ at in-trap pressures of up to 50 mTorr. It is clearly shown that high pressures can help to efficiently damp the axial KEs of the injected ions, including those with higher initial KE in addition to populations with wider KE distributions.
For 2D ion traps, the collision effects on ion trapping in the $x$ and $y$ dimensions are significantly different from that for the $\mathrm{z}$ dimension, since an rf field, instead of a DC field, is used to confine the ions in the $x-y$ plane. It is acknowledged that one of the main advantages for the 2D ion trap, if operated with balanced oppositephase rf, is the high efficiency of trapping externally injected ions, due to the injection being orthogonal to the rf field in the $x-y$ plane; hence, the result of such injections is a small initial KE in the $\mathrm{x}$ or $\mathrm{y}$ directions. However, the fringing field at the ends the ion trap could cause ion KE in the $x-y$ plane to increase significantly for an ion beam not perfectly of focused and collimated. For simpler versions of $2 \mathrm{D}$ ion trap mass spectrometers with a single-phase rf, the KE of the ions in the $x-y$ plane is expected to become significantly higher after entering the ion trap axially. The estimation of the trapping capability of the rf field in the $x-y$ plane needs to be made based on both pseudo potential well depth and collision effects. The collisions between the ions and molecules modify the ion kinetic energy and also randomize the ion motion directions; both processes have effects on the trapping efficiency. In this work, the maximum allowable ion kinetic energy in the $x-y$ plane was calculated for ions that can be trapped with an rf field at a given pressure. This can be used for characterizing the trapping capability of a $2 \mathrm{D}$ ion trap operated at a variety of pressures.

Using the pseudo potential approximation, $[1,50,51$, 59] the rf pseudo potential well depth for the rf trapping field in the $x-y$ plane of a 2D ion trap, at the absence of collisions with buffer gas, can be expressed by eq 3 :

$$
D_{u}=\frac{m q_{u}^{2} \Omega^{2} u_{0}^{2}}{16 z e}
$$

where $\Omega$ is the $\mathrm{rf}$ frequency, $q_{u}$ ( $\mathrm{u}$ denoting $\mathrm{x}$ or $\mathrm{y}$ ) is a Mathieu equation stability parameter $[1,59], \mathrm{u}_{0}$ is the ion trap dimension (from the $\mathrm{z}$-axis to the $\mathrm{x}$ or $\mathrm{y}$ electrodes), $\mathrm{m}$ is ion mass, $\mathrm{z}$ is the number of elemen-

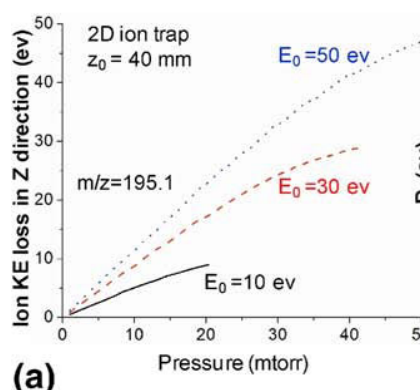

(a)

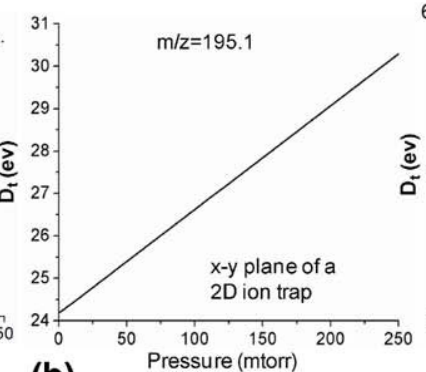

(b)

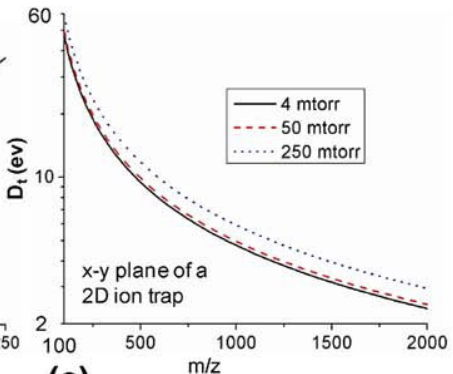

(c)

Figure 1. (a) Ion kinetic energy (KE) loss in the $\mathrm{z}$ direction for a $40 \mathrm{~mm}$ long $2 \mathrm{D}$ ion trap, calculated with an $80 \mathrm{~mm}$ path. The ions travel from the entrance end electrode to the back end electrode and are reflected back by the high potential at the back end electrode. $E_{0}$ represents the ion initial KE when it enters the ion trap. (b) Maximum allowable kinetic energy $\left(D_{t}\right)$ for ions to be trapped in the $x-y$ plane of a linear ion trap, as a function of pressure. (c) $D_{t}$ as a function of $m / z$ at pressures of 4,50 , and 250 mTorr. A constant rf voltage with a $\mathrm{q}_{\mathrm{u}}=0.4$ for $\mathrm{m} / \mathrm{z} 195.1$ was applied in a $2 \mathrm{D}$ ion trap $\left(x_{0}=y_{0}=5\right.$ $\mathrm{mm}, \mathrm{z}_{0}=40 \mathrm{~mm}$ ) with helium as the buffer gas and a $1.1 \mathrm{MHz} \mathrm{rf}$. 
tary charges on the ion, and $e$ is the elementary charge $\left(1.6 \times 10^{-19} \mathrm{C}\right)$. This model is expected to be valid at $q_{u}$ lower than 0.7 [59] and relatively more accurate $(<1 \%)$ results can be obtained with $q_{u}$ lower than 0.4 [20].

The maximum allowable KE $\left(D_{t}\right)$ in the $\mathrm{x}-\mathrm{y}$ plane for ions that can be trapped is determined by considering the combined effects of rf trapping and collisional damping. The formula for $D_{t}$ is shown in eq 4 (see Supplementary Material, which can be found in the electronic version of this article, for details):

$$
D_{t}=D_{u} \gamma^{-\frac{u_{0}}{\lambda}}
$$

The maximum allowable $\mathrm{KE}\left(D_{t}\right)$ in the $\mathrm{x}$-y plane of a $2 \mathrm{D}$ ion trap operated at a given pressure and $\mathrm{rf}$ amplitude was calculated for ion $\mathrm{m} / \mathrm{z} 195.1$ at different helium buffer gas pressures in a 2D linear ion trap with $x_{0}=y_{0}=5 \mathrm{~mm}$, as shown in Figure 1b. An rf frequency of $1.1 \mathrm{MHz}$ with an amplitude corresponding to $q_{u}=0.4$ for $\mathrm{m} / \mathrm{z} 195.1$, was used for the calculation. $D_{t}$ is shown to increase by $26 \%$ as the pressure increases from $1 \mathrm{mTorr}$ to $250 \mathrm{mTorr}$. $D_{t}$ was also calculated for ions over a mass range of up to $\mathrm{m} / \mathrm{z} 2000$ at helium pressures of 4, 50 and 250 mTorr with the same rf applied (Figure 1c). As expected, $D_{t}$ for large $\mathrm{m} / z$ ions is much shallower in comparison to ions of small $\mathrm{m} / \mathrm{z}$ due to the relatively lower $q_{u}$ values for the larger ions at the same rf voltage. The ion-neutral collisions can help to cool the ions, but using a higher rf amplitude to trap ions at a higher $q_{u}$ value generally has a more significant effect in improving the trapping efficiency. The estimation based on eq 4 is expected to be valid when the buffer gas damping force is much smaller than the rf trapping force. As discussed later in this paper (also see Supplementary Material for calculation details), under the given ion trap dimensions and rf parameters, these estimations will become invalid when the pressure is higher than 1 Torr.

Ions contained in an ion trap by the rf pseudopotential barrier will be concentrated toward the center region of the trap due to the damping effect of multiple collisions with buffer gas. However, the space charge effect, caused by the repulsion between same-polarity charged particles, results in the formation of an ion cloud with Gaussian distribution [60-63]. The improvement to the trapping well depth with increasing pressure is expected to enlarge the trapping capacity; however, the use of a 2D ion trap instead of a 3D ion trap seems to have a more significant effect. The ion cloud in a 2D ion trap has a cylindrical shape [64], while an ellipsoidally-shaped ion cloud occupies a 3D ion trap. A 40-times improvement in trapping capacity has been reported for a comparison between a $40 \mathrm{~mm}$ long 2D rectilinear ion trap $\left(x_{0}=5 \mathrm{~mm}, y_{0}=4 \mathrm{~mm}\right)$ and a $3 \mathrm{D}$ cylindrical ion trap $\left(r_{0}=z_{0}=5 \mathrm{~mm}\right)$ [64].

Helium gas is typically used in commercial ion trap mass spectrometers as a buffer gas while recently heavier gases such as $\mathrm{N}_{2}$ and air have also been used for linear ion traps $[16,32,65]$. It is highly preferable to use air as the buffer gas for portable instruments since no special gas supply is needed for field operations [66].
Helium and air have similar effects on ion cooling but a degradation of resolution has been observed with air, which can be improved by using nonlinear resonance ejection [32, 67]. Since air is mainly comprised of nitrogen $(78 \% ; 14.007 \mathrm{u})$ and oxygen $(21 \% ; 15.999 \mathrm{u})$, the kinetic energy loss of ions during collisions (eq 4) is expected to be bigger for air than for helium $(4.003 \mathrm{u})$. For example, the kinetic energy loss ratio $\gamma$ for ions of $\mathrm{m} / \mathrm{z} 195.1$ is 0.96 with helium but $\sim 0.75$ with air as the buffer gas. For heavier ions, the difference in the energy loss ratio between helium and air is much smaller due to the larger differences between the mass of the ion and the mass of the buffer gasses. For example, the kinetic energy loss ratio $\gamma$ for $\mathrm{m} / \mathrm{z} 2000$ is $\sim 0.996$ with helium as compared to $\sim 0.972$ with air as the buffer gas.

\section{Ion Resonance Ejection}

The mass analysis of ions trapped in an ion trap is most commonly done with a mass-selective instability scan, with which the rf amplitude is scanned linearly to cause the secular frequency of ions to increase until they become unstable [21]. A dipolar AC is usually applied to facilitate resonance ejection, which helps to significantly improve the ejection efficiency and the mass resolution [68, 69]. It is known that the optimal amplitude of the AC varies with changes of pressure, massto-charge ratio $(\mathrm{m} / \mathrm{z})$, and $\mathrm{AC}$ frequency [34, 64]. Though the general behavior of ions under dipolar AC excitation has been characterized previously with experiments [68], simulations [41], and calculations [45, 55], in this study modeling has been done to further understand the fundamental basis for the optimal conditions as well as to predict the resulted spectral qualities at these conditions.

\section{Ion Pseudo-Motion with AC Dipolar Excitation and Collision Effects}

Without considering the high order field effects, the motion of an ion trapped at $q_{u}<0.7$ can be approximated as a harmonic oscillator (also known as pseudo motion approximation [1]) with secular frequency $\omega_{0}$,

$$
d^{2} u / d t^{2}+\omega_{0}^{2} u=0
$$

With the presence of an AC dipolar excitation and ion-molecule collisions (modeled approximately by using the damping term), ion pseudo motion can be expressed using the time-dependent differential equation $[45-47,55,56,70]$

$$
\frac{d^{2} u}{d t^{2}}+c \frac{d u}{d t}+\omega_{0}^{2} u=f e^{j \omega_{s} t}
$$

where $\omega_{s}$ is the frequency of applied AC signal and $f=$ $\left(U_{a c} / 2 u_{0}\right)(e / m)$ with $U_{a c}$ as the amplitude of the applied AC. The value of the damping factor $c$ can be calculated based on Langevin collision theory [41, 49] with a hard sphere 
collision model (see Supplementary Material for details). The damping factor $c$ and the secular frequency $\omega_{0}$ can be used as the measures for the comparison between the buffer gas damping and pseudo-potential trapping force (see Supplementary Material for details). At an air buffer gas pressure of 1 Torr, the $\omega_{0}$ value is about 10 times the $c$ value for ion of $m / z 200\left(\sim 1.88 \times 10^{6}\right)$.

With consideration of the linear sweeping of the secular frequency during the mass-selective instability scan, eq 6 becomes

$$
\frac{d^{2} u}{d t^{2}}+c \frac{d u}{d t}+\left(\omega_{0}+a t\right)^{2} u=f e^{j \omega_{s} t}
$$

where $a$ is the ion secular frequency sweeping speed $\left(a=\frac{2 \sqrt{2 e}}{m z u_{0}^{2} \Omega} r f_{\text {rater }}\right.$, see Supplementary Material for derivation), which is directly related to the rf voltage scanning rate $\left(r f_{\text {rate }}\right)$. Following the treatments by Major and Dehmelt [51], Goeringer et al. [45], and Arnold et al. [55], the motion of a fast $u(t)$ oscillation can be expressed with the applied AC frequency $\left(\omega_{s}\right)$ and the slow varying motion $(U(t))$ as shown by following,

$$
u(t)=U(t) e^{j \omega_{s} t}
$$

Here, a linear approximation is used for the nonlinear differential equation; the linear approximation has been demonstrated to be effective for characterizing the envelope amplitude of the ion motion [45, 55]. By substituting eq 8 into eq 7 and ignoring the secondorder derivative of $U(t)$ (because $U(t)$ is a slow varying function with respect to time), eq 9 is obtained as

$$
\left(c+2 j \omega_{s}\right) \frac{d U}{d t}+\left[j c \omega_{s}-\omega_{s}^{2}+\left(\omega_{0}+a t\right)^{2}\right] U=f
$$

Solving eq 9 near resonance $\left(\omega_{s}-\omega_{0} \ll \omega_{s}\right.$, so that at $\left.\ll \omega_{s}\right)$ and ignoring the higher order terms of at, the envelope amplitude $U(t)$ of the ion motion is expressed as

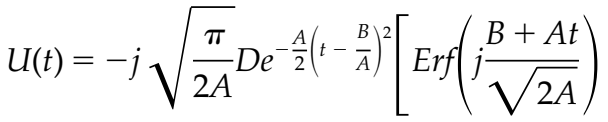

$$
\begin{aligned}
& \left.-\operatorname{Erf}\left(j \frac{B}{\sqrt{2 A}}\right)\right]+U(0) \\
& \text { with } A=\frac{2 a \omega_{0}}{c+2 j \omega_{s}}, B=\frac{j c \omega_{s}-\omega_{s}^{2}+\omega_{0}^{2}}{c+2 j \omega_{s}}, D=\frac{f}{c+2 j \omega_{s}}
\end{aligned}
$$

where Erf is the complex error function and $U(0)$ is the initial location of an ion at time zero. Using eq 10, the amplitude of ion secular motion can be calculated for ions during an rf voltage scan. The ion motion amplitudes for ion $m / z 195.1$ were calculated at pressures of $4.1,18.6$, and 48.3 mTorr (c values of 1957, 8878, and

23,054, respectively) and plotted as functions of the corresponding secular frequency (Figure 2a). An rf scan speed of $2797 \mathrm{Da} / \mathrm{s}$ and an AC signal of $294 \mathrm{kHz}$ and 2 $\mathrm{V}_{0-p}$ were used.

\section{Dipolar AC Resonance Ejection Optimization}

During a resonance excitation using a dipolar AC signal, the ion motion changes with the rf scan from an equilibrium state to an excited-state and then back to equilibrium state. As shown in Figure 2a, the corresponding frequency of the highest ion motion amplitude is not necessarily the same as the AC excitation frequency. At the same AC frequency and voltage, lower ion motion amplitudes were observed at higher pressures. When the ion motion amplitude is equal to or larger than the ion trap dimensions, the ions are considered to be ejected. A large number $(>5000)$ of initial ion positions with a Gaussian distribution (with a mean $=0$, corresponding to the center of the $x-y$ plane) were used and the corresponding ion ejection times were calculated to produce simulated mass spectra (Figure 2b; see Supplementary Material for detailed description). At a pressure of 4.1 mTorr, both a shift of the spectral peaks and a change of the peak widths were observed to occur when the AC voltage was varied. Hence, the frequency and the voltage of the dipolar $\mathrm{AC}$ need to be optimized to give the best mass resolution [34, 64].

The following approach was taken to understand the optimal conditions for resonance ejection. Eq 10 can be simplified as

$$
U(t)=g(t)+U(0)
$$

where $\mathrm{g}(\mathrm{t})$ represents $-j \sqrt{\frac{\pi}{2 A}} D e^{-\frac{A}{2}\left(t-\frac{B}{A}\right)^{2}}\left[\operatorname{Erf}\left(j \frac{B+A t}{\sqrt{2 A}}\right)-\right.$ $\left.\operatorname{Erf}\left(j \frac{B}{\sqrt{2 A}}\right)\right]$ for simplification. A peak's width in a mass spectrum is due to the differences of the initial ion positions. Therefore, the ejection times of two ions $\left(t_{1}\right.$ and $\left.t_{2}\right)$ with the same $m / z$ value but different initial positions $\left[U^{\prime}(0)\right.$ and

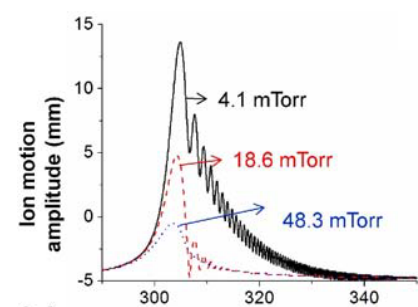

(a)

Ion secular frequency $(\mathrm{kHz})$
Figure 2. Pressure effect on ion motion with an AC dipolar excitation for resonance ejection. (a) Ion motion amplitude as a function of the secular frequency during an rf scan. (b) Calculated mass spectrum for $m / z 195.1$ with AC dipolar excitation at different voltages. Operating parameters: $\mathrm{AC}$ frequency $=303 \mathrm{KHz}, \mathrm{rf}$ scan rate $=2797 \mathrm{~m} / \mathrm{z} / \mathrm{s}$, and air as the buffer gas. 
$\left.U^{\prime \prime}(0)\right]$ from an ion trap (with dimension $u_{0}$ ) can be calculated as

$$
t_{1}=g^{-1}\left(u_{0}-U^{\prime}(0)\right), \quad t_{2}=g^{-1}\left(u_{0}-U^{\prime \prime}(0)\right)
$$

The difference between the ejection times of these two ions is then

$$
\begin{aligned}
& t_{2}-t_{1}=g^{-1}\left(U_{2}\right)-g^{-1}\left(U_{1}\right) \\
& \text { with } U_{2}=u_{0}-U^{\prime \prime}(0), U_{1}=u_{0}-U^{\prime}(0)
\end{aligned}
$$

Assuming that the ejection time difference is small, the ejection time difference is thus proportional to the slope of the function $g^{-1}(U)$.

$$
\frac{d t}{d U(0)}=\frac{t_{2}-t_{1}}{U_{2}-U_{1}}=\frac{g^{-1}\left(U_{2}\right)-g^{-1}\left(U_{1}\right)}{U_{2}-U_{1}}=\frac{d}{d U^{d}} g^{-1}(U)
$$

To obtain the highest resolution, all the ions of the same $\mathrm{m} / \mathrm{z}$ value must eject within a time period which is as narrow as possible. According to eq 14, the minimal ejection time difference is obtained at a minimum value of $\frac{d}{d U} g^{-1}(U)$, which corresponds to a maximum value of $\frac{d}{d t} g(t)$; physically, this minimization of the distribution of ejection times occurs when the ions are ejected at the point where the ion motion amplitude $(g(t))$ increases at the highest rate during the dipolar excitation.

The initial positions of the ions at the beginning of the rf scan with AC excitation can be described using Gaussian distributions with a mean of 0 corresponding to the center of the $\mathrm{x}-\mathrm{y}$ plan of a $2 \mathrm{D}$ ion trap. Different standard deviations (std) of the Gaussian distribution can be used to describe the different sizes of the ion clouds. For each specific case with a unique set of initial ion positions at a given pressure, the spectral peak width can be calculated for each of the excitation AC amplitudes using the method described above. The full widths at half maximum (FWHM) calculated at 4.1 Torr for ions of $m / z 195.1$ with different initial ion cloud sizes ( $\mathrm{std}=0.15 \mathrm{x}_{0}, 0.237 \mathrm{x}_{0}$, and $0.3 \mathrm{x}_{0}$ ) are plotted in Figure 3a. The optimization curves are similar to what was observed with the optimization of the AC excitation during experiments [34]. Though the resolution for resonance ejection became worse as the initial ion position distribution was widened, the optimized resonance AC voltages, corresponding to the minimum FWHM of each curve, were found to be the same at a given pressure. In all, the FWHMs were calculated for optimizing the resonance AC amplitude at pressures from 4.1 to 250 mTorr. The optimization curves for pressures of 4.1, 18.6, and 48.3 mTorr are plotted in Figure $3 \mathrm{~b}$. It was observed that the minimum peak width increases at higher pressures, and higher AC amplitudes are required to obtain the best resolution at higher pressures. The optimal AC voltages required for obtaining the best mass resolution for $m / z 195.1$ were found from the optimization curves and plotted as a function of pressure, as shown in Figure 3c. The data obtained from experimental characterization [34] for pressures of up to 48.3 mTorr are also plotted in Figure 3c. Clearly, the experimental and calculated results match well for this pressure range.

\section{Mass Resolution at High Pressures}

Attempts have been made to predict the mass resolution (FWHM) for ion traps operated at high pressures with optimized AC resonance ejection using the method described above (eq 10). To use the experimental data to validate the calculated data for prediction, the operating conditions used for the experimental characterization were estimated and used in the calculation. The ion cloud expansion under equilibrium $(U(0))$ used in the calculation was adjusted by matching the calculated and experimentally obtained FWHM at a pressure of 4.1 $\mathrm{mTorr}$ for $\mathrm{m} / \mathrm{z}$ 195.1. The estimated ion cloud expansion (the radius of the ion cloud cylinder in a $2 \mathrm{D}$ trap) is about $0.237 \times u_{0}$, which is about $1.185 \mathrm{~mm}$ for a $2 \mathrm{D}$ trap with $x_{0}=y_{0}=5 \mathrm{~mm}$. Using this estimated ion cloud expansion, the mass resolution for $m / z 195.1$ was calculated over a pressure range of 4.1 to 250 mTorr (Figure
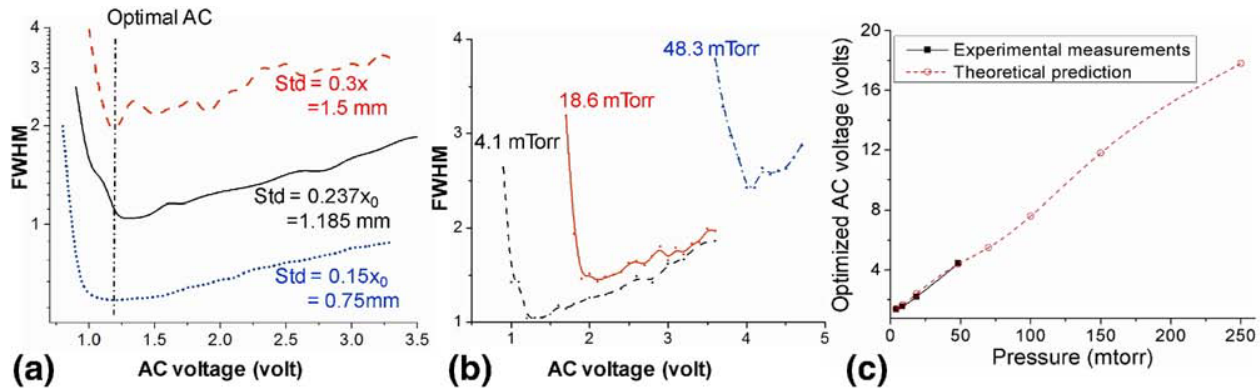

Figure 3. Optimization of AC dipolar resonance ejection. (a) Peak width as a function of AC voltage with different initial ion cloud sizes. (b) Peak width as a function of the AC voltage at different pressures. (c) Predicted optimal voltage of AC dipolar excitation for resonance ejection of $m / z 195.1$ as a function of pressure. Operating parameters: AC frequency $=303 \mathrm{KHz}$, rf scan rate $=2797 \mathrm{~m} / \mathrm{z} / \mathrm{s}$, and air as the buffer gas. 
4). The semiempirical peak resolutions for pressures below 48.3 mTorr (Figure 4a) matched well with those obtained from experimental characterization (Figure $4 b$ ). On this basis, a mass resolution better than $\mathrm{m} / \mathrm{z} 9.5$ (FWHM) is predicted for pressures below $250 \mathrm{mTorr}$ with optimized resonance ejection.

\section{Ion Isolation}

Mass-selective ion isolation is a critical step in tandem mass spectrometry (MS/MS). A variety of different methods have been developed for ion isolation in ion traps $[1,71]$, with the notched SWIFT (stored waveform inverse Fourier transform) method [11, 34, 72] being the most commonly used today. Unlike dipolar AC ejection in an ion trap, SWIFT excites and ejects unwanted ions via a broadband waveform; the targeted ions are retained if their secular frequencies are within the notches of the waveform. The efficiency of ion isolation is measured by the percentage of the targeted ions that can be isolated with a given $\mathrm{m} / \mathrm{z}$ window [64, 65,73]. Ideally, ions with the same $\mathrm{m} / \mathrm{z}$ have the same secular frequency (a $\Delta$ function in the frequency spectrum), and a $100 \%$ isolation efficiency can be achieved with an ultra narrow notch in the isolation waveform. However, secular frequency distribution of ions at the same $\mathrm{m} / \mathrm{z}$ can be broadened as a result of ion-molecule collisions, space charge, and imperfect electric filed, etc $[44,45,64$, 67]; hence, the ions nominally placed in the notch of the isolation waveform may also be excited. Therefore, it is common practice to broaden the notches so that a majority (or all) of the targeted ions are not ejected (i.e., high isolation efficiency may require poor isolation resolution).

Physically, the isolation efficiency is highly dependent on the distribution of the response (ion motion amplitude) of the ions within a secular frequency range at the vicinity of the frequency $\omega_{s}$ of an excitation AC signal. The response distribution of the targeted ions can be experimentally characterized using methods previously described [61, 62]. In these methods, a dipolar excitation AC is applied and the response of the ion motion amplitude is measured indirectly while the rf voltage is set to a value corresponding to a secular frequency of the targeted ions.

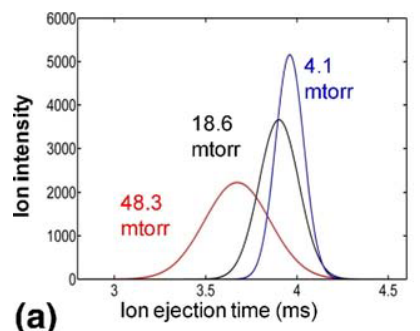

(a)

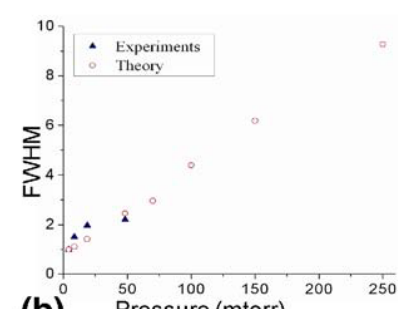

(b) Pressure (mtorr)

Figure 4. (a) Peak broadening at high pressures with optimized resonance ejection conditions. (b) Predicted resolution for mass analysis of $\mathrm{m} / \mathrm{z} 195.1$ as a function of pressure. Operating parameters: $\mathrm{AC}$ frequency $=303 \mathrm{KHz}, \mathrm{rf}$ scan rate $=2797 \mathrm{~m} / \mathrm{z} / \mathrm{s}$, and air as the buffer gas.
Based on the theoretical method developed here, the pressure effects on the excitation response distribution can also be estimated. During an ion isolation process, the ion secular frequency remains constant, so the ion secular frequency sweeping speed $a$ in eqs 7 and 9 should be set to zero. Following a derivation procedure similar to eq 10 (see Supplementary Material for details), the ion motion amplitude at a secular frequency $\omega_{0}$ in response to dipolar excitation at $\omega_{s}$ can be calculated by

$$
\begin{aligned}
& U(t)=\frac{f}{M}+K_{0} e^{-N t} \\
& \text { with } M=j c \omega_{s}-\omega_{s}^{2}+\omega_{0}^{2}, N=\frac{M}{c+2 j \omega_{s}}
\end{aligned}
$$

where $K_{0}$ is determined by the ion initial condition and is equal to $-f / M$ if the ion is cooled to the center of the trap before AC excitation is applied.

It has been previously shown that ion motion in response to an off-resonance dipolar AC excitation becomes stable within $5 \mathrm{~ms}$ when the pressure is above 1 mTorr [11]. In this study, the ion motion amplitude $U(t)$ was calculated for each AC excitation at a given pressure from time 0 to $5 \mathrm{~ms}$ (with 1000 steps) and the highest amplitude was recorded as the excitation response, which determines if the ions are ejected or not.

Figure 5a shows the excitation response distributions for $m / z 195.1$ at $4.1,18.6$, and 48.3 mTorr with a dipolar excitation of $1 \mathrm{~V}_{0-p}$ at frequency $\omega_{s}=303 \mathrm{kHz}$. The excitation responses were first calculated using eq 15 for each of the ion secular frequencies (determined by the rf voltage) at different pressures and then normalized by the highest value. The response distribution, which also represents the secular frequency spread of the ions, is significantly broadened at higher pressures due to intensive ion-molecule collisions. For isolation using a notched waveform, it is desirable for the target ions with secular frequencies within the notch to have minimum excitation response for frequencies at (or near) the notch periphery; however, such unwanted resonance is more likely at higher pressures due to broader secular frequency distributions. As such, for high-pressure isolation, it may be difficult to achieve high efficiency SWIFT isolation with narrow isolation windows.

At a given pressure, the isolation efficiency for ions with a nominal secular frequency centered in an isolation window can be calculated by integrating the number of the ions from the secular frequency distribution which reside within the isolation window. The minimum notch widths for isolating ions of $\mathrm{m} / z 195.1$ (at $\omega_{0}=303 \mathrm{kHz}$ ) at a percentage of $95.4 \%, 84.3 \%$, and $52.1 \%$ were calculated over a pressure range of 1 to 250 mTorr (Figure $5 b)$. Since the rf field in the $x-y$ plane of a 2D ion trap is identical to that of a mass filter, the isolation window width $\Delta(\mathrm{m} / \mathrm{z})$ can be calculated from $\Delta \omega_{0}$ using the 
following equations (see Supplementary Material for additional information) $[20,71,74]$ :

$$
\triangle(m / z)=\frac{(m / z)}{q_{u}} \frac{4 \triangle \omega_{0} \beta}{\Omega\left(\frac{2 q_{u}}{2-q_{u}^{2}}+\frac{2 q_{u}^{3}}{\left(2-q_{u}^{2}\right)^{2}}-\frac{3}{8} q_{u}^{3}+\frac{29}{384} q_{u}^{5}\right)}
$$

where

$$
\beta=\sqrt{\frac{q_{u}^{2}}{2-q_{u}^{2}}+\frac{3 q_{u}^{4}}{32}+\frac{29 q_{u}^{6}}{2304}}=\frac{2 \omega_{0}}{\Omega}
$$

As expected, the required isolation window width is much wider to achieve a higher isolation percentage at higher pressure. An isolation window of about $\Delta(\mathrm{m} / \mathrm{z})=$ 8 allows $52.1 \%$ of the ions at $m / z 195.1\left(\omega_{0}=303 \mathrm{kHz}\right)$ to be isolated at 250 mTorr.

The $m / z$ dependence of the minimum isolation window width for a given isolation percentage has also been studied. The minimum isolation window required for isolating $m / z 195.1$ at a targeted isolation efficiency (for example, $84.3 \%$ ) at a specific pressure can be easily obtained based on the data shown in Figure 5b. This procedure was performed for other ions, within a mass range up to $m / z 2000$ and with a targeted isolation efficiency of $84.3 \%$, at pressures of $1,10,20$, and 50 mTorr. The minimum isolation windows are plotted as a function of the $m / z$ value for each of the pressures (Figure 5c). At low buffer gas pressure, broader isolation windows are required for high- $m / z$ ions to achieve the same isolation efficiency as that of low- $m / z$ ions. As the pressure increases, this $m / z$ effect becomes less apparent. At a pressure near 50 mTorr, the same isolation window can be used for ions over the tested mass range to achieve similar isolation efficiencies, that is, the collision effect on ion isolation efficiency becomes overwhelming. Mathematically, the isolation window width is a complex function of the mass-to-charge ratio and the damping term $c$, which is indirectly represented by the term $\Delta \omega_{0}$ in eq 16. At high-pressure, based on the hard sphere collision model, collisions have less effect on the motion of heavier ions. For example, the value of the damping term $c$ at 50 mTorr is 23,893 for ion $\mathrm{m} / \mathrm{z}$ 195.1 but 2350 for ion $\mathrm{m} / \mathrm{z}$ 2000; the increasing trend in the ion isolation window as a function of $\mathrm{m} / \mathrm{z}$ is compensated by the decreasing trend in the damping term due to the collision effect.

\section{Conclusions}

In this study, an attempt has been made to use theoretical models to better understand the collision effects in ion trap mass analysis and to predict trap performance at pressures higher than that which can currently be characterized experimentally. The theoretical method used here is expected to be valid for buffer gas pressures as high as 1 Torr. For the Langevin collision theory used in the method, the ion-molecule collision probability is calculated based on the assumption that the interacting ion-molecule pair is isolated from other molecules; in other words, the distance between gasphase particles is larger than the collision cross section diameter for ion-molecule collisions. At 1 Torr, based on the ideal gas law for a model system with air molecules and ions of $m / z 200$ with 1 eV kinetic energy, the distance between the molecules is about 40 times the ion-molecule collision cross section diameter.

In practice, it is reasonable to expect an ion trap to perform mass-selective operations at several hundred mTorr, though at relatively poor resolution. When an ion trap or other rf-driven device operates at highpressure, its analytical effectiveness is dependent on an appropriate balance between the effects of the electric field and ion-neutral collisions. For a 2D ion trap of $u_{0}=5 \mathrm{~mm}$ operating with $1.1 \mathrm{MHz}$ rf at 1 Torr, the force due to the electric field on ions of $\mathrm{m} / \mathrm{z} 200$ with a nominal secular frequency of $300 \mathrm{kHz}$ is about 10 times the force due to collisions with air buffer. The effectiveness of ion confinement and focusing by an rf field has been demonstrated by ion funnels working at 1 Torr [75]. The rf force under the above conditions will no

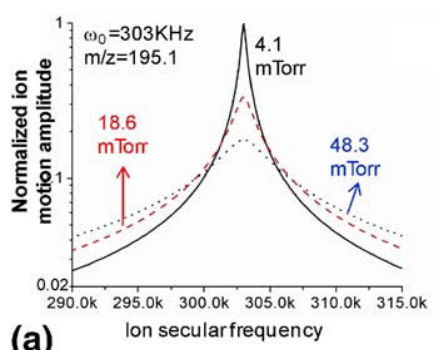

(a)

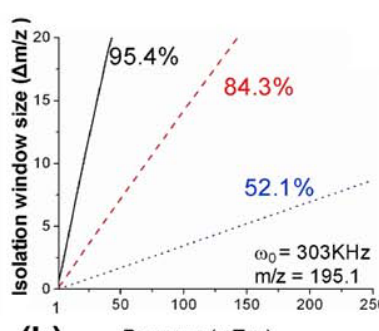

(b)

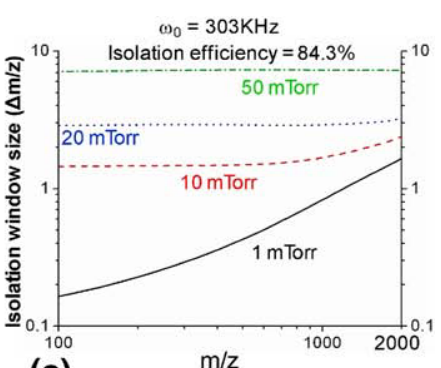

(c)

Figure 5. Pressure effects on ion isolation. (a) Ion secular frequency broadening at different pressures; (b) Required isolation window width for the specified isolation efficiencies as functions of pressure. (c) Required isolation window width for an $84 \%$ ion isolation efficiency over the mass range of $\mathrm{m} / \mathrm{z} 100$ to 2000 at various pressures. Operating parameters: AC frequency $=303 \mathrm{KHz}$, $\mathrm{rf}$ scan rate $=2797$ $\mathrm{m} / \mathrm{z} / \mathrm{s}$, and air as the buffer gas. 
longer dominate the ion motion when the pressure becomes higher than 1 Torr.

\section{Acknowledgments}

The authors acknowledge support for this work by the NSF Sensors and Sensor Networks Grant, award no. SST: 0528948 and the National Science Foundation of China (project no. 20728505).

\section{Appendix A Supplementary Material}

Supplementary material associated with this article may be found in the online version at doi:10.1016/ j.jasms.2009.06.019.

\section{References}

1. March, R. E.; Londry, F. A. Theory of Quadrupole Mass Spectrometry. In Practical Aspects of ion tap mass spectrometry; Vol. I. March, R. E., Todd, J. F. J., Eds.; CRC: Boca Raton, FL, 1995; pp. 25.

2. Richard, G. S. Transport of ions at atmospheric pressure using an electrostatic surface wave. J. Appl. Phys. 1989, 66, 2253-2259.

3. Lin, B.; Sunner, J. Ion transport by viscous gas flow through capillaries. J. Am. Soc. Mass Spectrom. 1994, 5, 873-885.

4. Lock, C. M.; Dyer, E. W. Simulation of ion trajectories through a high pressure radio frequency only quadrupole collision cell by SIMION 6.0. Rapid Commun. Mass Spectrom. 1999, 13, 422-431.

5. Wu, G.; Cooks, R. G.; Ouyang, Z.; Yu, M.; Chappell, W. J.; Plass, W. R. Ion Trajectory Simulation for Electrode Configurations with Arbitrary Geometries. J. Am. Soc. Mass Spectrom. 2006, 17, 1216-1228.

6. Kim, T.; Tolmachev, A. V.; Harkewicz, R.; Prior, D. C.; Anderson, G.; Udseth, H. R.; Smith, R. D.; Bailey, T. H.; Rakov, S.; Futrell, J. H. Design and Implementation of a New Electrodynamic Ion Funnel. Anal. Chem. 2000, 72, 2247-2255

7. Fico, M.; Maas, J. D.; Smith, S. A.; Costa, A. B.; Chappell, W. J.; Cooks, R. G. Circular Arrays of Polymer-based Miniature Rectilinear Ion Traps; (submitted 2009), unpublished.

8. Schwartz, J. C.; Syka, J. E. P.; Quarmby, S. T. Thermo Finnigan, San Jose, CA, U.S. patent, 0142705; 2008.

9. Xia, Y.; Thomson, B. A.; McLuckey, S. A. Bidirectional Ion Transfer between Quadrupole Arrays: MS $^{\mathrm{n}}$ Ion/Ion Reaction Experiments on a Quadrupole/Time-of-Flight Tandem Mass Spectrometer. Anal. Chem. 2007, 79, 8199-8206

10. Makarov, A.; Denisov, E.; Kholomeev, A.; Balschun, W.; Lange, O.; Strupat, K.; Horning, S. Performance Evaluation of a Hybrid Linear Ion Trap/Orbitrap Mass Spectrometer. Anal. Chem. 2006, 78, 2113-2120.

11. Song, Q.; Smith, S. A.; Gao, L.; Xu, W.; Volný, M.; Zheng, O.; Cooks, R. G. Mass Selection of Ions from Beams Using Waveform Isolation in Radiofrequency Quadrupoles. Anal. Chem. 2009, 81, 1833-1840.

12. Blake, T. A.; Ouyang, Z.; Wiseman, J. M.; Takats, Z.; Guymon, A. J.; Kothari, S.; Cooks, R. G. Preparative Linear Ion Trap Mass Spectrometer for Separation and Collection of Purified Proteins and Peptides in Arrays Using Ion Soft Landing. Anal. Chem. 2004, 76, 6293-6305.

13. Ouyang, Z.; Takats, Z.; Blake, T. A.; Gologan, B.; Guymon, A. J.; Wiseman, J. M.; Oliver, J. C.; Davisson, V. J.; Cooks, R. G. Preparing Protein Microarrays by Soft-Landing of Mass-Selected Ions. Science 2003, 301, 1351-1354.

14. Patterson, G. E.; Knecht, B. A. Griffin Analytical Technologies: U.S. patent, 7161142; 2007.

15. Yang, M.; Kim, T.-Y.; Hwang, H.-C.; Yi, S.-K.; Kim, D.-H. Development of a Palm Portable Mass Spectrometer. J. Am. Soc. Mass Spectrom. 2008, $19,1442-1448$

16. Gao, L.; Sugiarto, A.; Harper, J. D.; Cooks, R. G.; Ouyang, Z. Design and Characterization of a Multisource Hand-Held Tandem Mass Spectrometer. Anal. Chem. 2008, 80, 7199-7205.

17. Austin, D. E.; Peng, Y.; Hansen, B. J.; Miller, I. W.; Rockwood, A. L.; Hawkins, A. R.; Tolley, S. E. Novel Ion Traps Using Planar Resistive Electrodes: Implications for Miniaturized Mass Analyzers. J. Am. Soc. Mass Spectrom. 2008, 19, 1435-1441.

18. Contreras, J. A.; Murray, J. A.; Tolley, S. E.; Oliphant, J. L.; Tolley, H. D.; Lammert, S. A.; Lee, E. D.; Later, D. W.; Lee, M. L. Hand-Portable Gas Chromatograph-Toroidal Ion Trap Mass Spectrometer (GC-TMS) for Detection of Hazardous Compounds. J. Am. Soc. Mass Spectrom. 2008, $19,1425-1434$

19. Gao, L.; Cooks, R. G.; Ouyang, Z. Breaking the Pumping Speed Barrier in Mass Spectrometry: Discontinuous Atmospheric Pressure Interface. Anal. Chem. 2008, 80, 4026-4032.

20. Wuerker, R. F.; Goldenberg, H. M.; Langmuir, R. V. Electrodynamic Containment of Charged Particles by Three-Phase Voltages. J. App. Phys. 1959, 30, 441-442.
21. Stafford, G. C.; Kelley, P. E.; Syka, J. E. P.; Reynolds, W. E.; Todd, J. F. J Recent improvements in and analytical applications of advanced ion trap technology. Int. J. Mass Spectrom. Ion Processes 1984, 60, 85-98.

22. Louris, J. N.; Amy, J. W.; Ridley, T. Y.; Cooks, R. G. Injection of ions into a quadrupole ion trap mass spectrometer. Int. J. Mass Spectrom. Ion Processes 1989, 88, 97-111.

23. Douglas, D. J.; French, J. B. Collisional focusing effects in radio frequency quadrupoles. J. Am. Soc. Mass Spectrom. 1992, 3, 398-408.

24. Wu, H.-F.; Brodbelt, J. S. Effects of reactant ion kinetic energy on both endothermic and exothermic ion/molecule reactions in a quadrupole ion trap mass spectrometer. Int. J. Mass Spectrom. Ion Processes 1993, 124, 175-184.

25. Holzscheiter, M. H. Cooling of Particles Stored in Electromagnetic Traps. Physica Scripta 1988, T22, 73-78

26. Kofel, P. In Practical Aspects of Ion Trap Mass Spectrometry, Vol. II, March, R. E., Todd, J. F. J., Eds.; CRC: Boca Raton, FL, 1995; pp 51-88.

27. Cooks, R. G. Special feature: Historical Collision-induced dissociation: Readings and commentary. J. Mass Spectrom. 1995, 30, 1215-1221.

28. Dodonov, A.; Kozlovsky, V.; Loboda, A.; Raznikov, V.; Sulimenkov, I.; Tolmachev, A.; Kraft, A.; Wollnik, H. A new technique for decomposition of selected ions in molecule ion reactor coupled with ortho time-of-flight mass spectrometry. Rapid Commun. Mass Spectrom. 1997, $11,1649-1656$.

29. Javahery, G.; Thomson, B. A segmented radiofrequency-only quadrupole collision cell for measurements of ion collision cross section on a triple quadrupole mass spectrometer. J. Am. Soc. Mass Spectrom. 1997, 8 , 697-702.

30. Mansoori, B. A.; Dyer, E. W.; Lock, C. M.; Bateman, K.; Boyd, R. K.; Thomson, B. A. Analytical performance of a high-pressure radio frequency-only quadrupole collision cell with an axial field applied by using conical rods. J. Am. Soc. Mass Spectrom. 1998, 9, 775-788.

31. Mitchell Wells, J.; McLuckey, S. A.; Burlingame, A. L. In Methods in Enzymology, Vol. CDII, Academic Press: San Diego, CA, 2005; pp 148-185.

32. Gao, L.; Song, Q.; Patterson, G. E.; Cooks, R. G.; Ouyang, Z. Handheld Rectilinear Ion Trap Mass Spectrometer. Anal. Chem. 2006, 78, 59946002 .

33. Ouyang, Z.; Wu, G.; Song, Y.; Li, H.; Plass, W. R.; Cooks, R. G. Rectilinear Ion. Trap: Concepts, Calculations, and Analytical Performance of a New Mass Analyzer. Anal. Chem. 2004, 76, 4595-4605.

34. Song, Q.; Xu, W.; Gao, L.; Smith, S. A.; Cooks, R. G.; Ouyang, Z. Mass Analysis at High Pressure Using Ion Trap: Experimental Characterization, unpublished (submitted).

35. Mitchell, D. W. Realistic simulation of the ion cyclotron resonance mass spectrometer using a distributed three-dimensional particle-in-cell code. J. Am. Soc. Mass Spectrom. 1999, 10, 136-152.

36. Nikolaev, E. N.; Heeren, R. M. A.; Popov, A. M.; Pozdneev, A. V.; Chingin, K. S. Realistic modeling of ion cloud motion in a Fourier transform ion cyclotron resonance cell by use of a particle-in-cell approach. Rapid Commun. Mass Spectrom. 2007, 21, 3527-3546.

37. Wu, G.; Cooks, R. G.; Ouyang, Z. Geometry optimization for the cylindrical ion trap: Field calculations, simulations, and experiments. Int. J. Mass Spectrom. 2005, 241, 119-132.

38. Ding, L.; Sudakov, M.; Kumashiro, S. A simulation study of the digital ion trap mass spectrometer. Int. J. Mass Spectrom. 2002, 221, 117-138.

39. Bui, H. A.; Cooks, R. G. Windows version of the ion trap simulation program ITSIM: A powerful heuristic and predictive tool in ion trap mass spectrometry. J. Mass Spectrom. 1998, 33, 297-304.

40. Salazar, G. A.; Masujima, T. Computer Simulation of the Gap-Tripole Ion Trap with Linear Injection, 3D Ion Accumulation, and Versatile Packet Ejection. J. Am. Soc. Mass Spectrom. 2008, 19, 1367-1374.

41. Londry, F. A.; Alfred, R. L.; March, R. E. Computer simulation of single-ion trajectories in Paul-type ion traps. J. Am. Soc. Mass Spectrom. 1993, 4, 687-705.

42. McLachlan, N. W. Theory and Application of Mathieu Functions, Clarendon Press: Oxford, 1947; pp 1-402.

43. Comisarow, M. B.; Marshall, A. G. Fourier transform ion cyclotron resonance spectroscopy. Chem. Phys. Lett. 1974, 25, 282-283.

44. Wang, Y.; Franzen, J.; Wanczek, K. P. The nonlinear resonance ion trap. Part 2. A general theoretical analysis. Int. J. Mass Spectrom. Ion Processes 1993, 124, 125-144.

45. Goeringer, D. E.; Whitten, W. B.; Ramsey, J. M. Theory of highresolution mass spectrometry achieved via resonance ejection in the quadrupole ion trap. Anal. Chem. (Washington) 1992, 64, 1434-1439.

46. Makarov, A. A. Resonance Ejection from the Paul Trap: A Theoretica Treatment Incorporating a Weak Octopole Field. Anal. Chem. 1996, 68 , 4257-4263.

47. Whitten, W. B.; Reilly, P. T.; Ramsey, J. M. High-pressure ion trap mass spectrometry. Rapid Commun. Mass Spectrom. 2004, 18, 1749-1752.

48. Xu, W.; Chappell, W. J.; Cooks, G. R.; Ouyang, Z. Characterization of electrode surface roughness and its impact on ion trap mass analysis. $J$. Mass Spectrom., unpublished (corrected proof).

49. George, G.; Stevenson, D. P. Reactions of Gaseous Molecule Ions with Gaseous Molecules. V. Theory. J. Chem. Phys. 1958, 29, 294-299.

50. Wuerker, R. F.; Shelton, H.; Langmuir, R. V. Electrodynamic Containment of Charged Particles. J. Appl. Phys. 1959, 30, 342-349.

51. Major, F. G.; Dehmelt, H. G. Exchange-Collision Technique for the rf Spectroscopy of Stored Ions. Phys. Rev. 1968, 170, 91-107.

52. Plass, W. R.; Cooks, R. G. A model for energy transfer in inelastic molecular collisions applicable at steady state or non-steady state and 
for an arbitrary distribution of collision energies. J. Am. Soc. Mass Spectrom. 2003, 14, 1348-1359.

53. Whetten, N. R. Macroscopic particle motion in quadrupole fields. J. Vacuum Sci. Tech. 1974, 11, 515-518.

54. Dawson, P. H.; Whetten, N. R. Non-linear resonances in quadrupole mass spectrometers due to imperfect fields. I. The quadrupole ion trap. Int. J. Mass Spectrom. Ion Phys. 1969, 2, 45-59.

55. Arnold, N. S.; Hars, G.; Meuzelaar, H. L. C. Extended theoretical considerations for mass resolution in the resonance ejection mode of quadrupole ion trap mass spectrometry. J. Am. Soc. Mass Spectrom. 1994, $5,676-688$

56. Zhao, X. Granot, O.; Douglas, D. J. Quadrupole Excitation of Ions in Linear Quadrupole Ion Traps with Added Octopole Fields. J. Am. Soc. Mass Spectrom. 2008, 19, 510-519.

57. Raymond, D. http://physics.nmt.edu/ raymond/classes/ph13xbook/ node107.html, 2006.

58. Senko, M. W.; Schwartz, J. C. Trapping Efficiency Measurements in a 2D Ion Trap Mass Spectrometer. Proceedings of the 50th ASMS Conference; Orlando, FL, June, 2002

59. Hoffmann, E. D.; Stroobant, V. Mass Spectrometry: Principles and Applications, 2nd ed.; John Wiley and Sons: New York, 2002; pp 63-83.

60. Knight, R. D.; Prior, M. H. Laser scanning measurement of the density distribution of confined $6 \mathrm{Li}+$ ions. J. Appl. Phys. 1979, 50, 3044-3049.

61. Hemberger, P. H.; Nogar, N. S.; Williams, J. D.; Cooks, R. G.; Syka, J. E. P. Laser photodissociation probe for ion tomography studies in a quadrupole ion-trap mass spectrometer. Chem. Phys. Lett. 1992, 191, 405-410.

62. Williams, J. D.; Cooks, R. G.; Syka, J. E. P.; Hemberger, P. H.; Nogar, N. S. Determination of positions, velocities, and kinetic energies of resonantly excited ions in the quadrupole ion trap mass spectrometer by layer photodissociation. J. Am. Soc. Mass Spectrom. 1993, 4, 792-797.

63. Lammert, S. A.; Cleven, C. D.; Cooks, R. G. Determination of ion frequencies in a quadrupole ion trap by using a fast direct current pulse as pump and a laser probe. J. Am. Soc. Mass Spectrom. 1994, 5, $29-36$.
64. Schwartz, J. C.; Senko, M. W.; Syka, J. E. P. A two-dimensional quadrupole ion trap mass spectrometer. J. Am. Soc. Mass Spectrom. 2002 $13,659-669$.

65. James, W. H. A new linear ion trap mass spectrometer. Rapid Commun. Mass Spectrom. 2002, 16, 512-526.

66. Ouyang, Z.; Noll, R. J.; Cooks, R. G. Handheld Miniature Ion Trap Mass Spectrometers. Anal. Chem. 2009, 81, 2421-2425.

67. Lammert, S. A.; Wells, J. M. Experimental investigation into the performance of ion traps using air versus helium as the buffer gas. Rapid Commun. Mass Spectrom. 1996, 10, 361-371.

68. Stafford, G. C.; Kelley, P. E.; Stephens, D. R. Finnigan Corporation: U.S patent, 4540884; 1985

69. Fulford, J. E.; Nhu-Hoa, D.; Hughes, R. J.; March, R. E.; Bonner, R. F. Wong, G. J. Radio-frequency mass selective excitation and resonant ejection of ions in a three-dimensional quadrupole ion trap. J. Vac. Sci. Technol. 1980, 17, 829-835.

70. Sudakov, M.; Douglas, D. J. Linear quadrupoles with added octopole fields. Rapid Commun. Mass Spectrom. 2003, 17, 2290-2294.

71. Yost, R. A.; Enke, C. G. Selected ion fragmentation with a tandem quadrupole mass spectrometer J. Am. Chem. Soc. 1978, 100, 2274-2275.

72. Soni, M. H. Cooks, R. G. Selective injection and isolation of ions in quadrupole ion trap mass spectrometry using notched waveforms created using the inverse Fourier transform. Anal. Chem. (Washington), 1994, 66(15), 2488-2496.

73. Song, Q.; Kothari, S.; Senko, M. A.; Schwartz, J. C.; Amy, J. W.; Stafford G. C.; Cooks, R. G.; Ouyang, Z. Rectilinear Ion Trap Mass Spectromete with Atmospheric Pressure Interface and Electrospray Ionization Source. Anal. Chem. 2006, 78, 718-725.

74. Dawson, P. H. Quadrupole Mass Spectrometry and its application; Elsevier Scientific Publishing Company: New York, 1976; pp 19, 20, 71.

75. Shaffer, S. A.; Tang, K.; Anderson, G. A.; Prior, D. C.; Udseth, H. R. Smith, R. D. A novel ion funnel for focusing ions at elevated pressure using electrospray ionization mass spectrometry. Rapid Commun. Mass Spectrom. 1997, 11, 1813-1817. 